\title{
Innovación y competitividad: dónde se ubica Latinoamérica compa- rativamente según los indicadores
}

\section{Innovation and competitiveness: Where Latin America is located comparatively according to the indicators}

\author{
Francisco Javier Quesada Espinoza ${ }^{1}$ \\ Universidad Estatal a Distancia, Costa Rica
}

\begin{abstract}
Resumen. Es común asociar la innovación con el nivel de desarrollo y competitividad de un país. El Índice Mundial de Innovación elabora una clasificación en este ámbito. Para los países latinoamericanos se estudió la producción de patentes, la influencia de las variables que contempla el índice en su evaluación y la clasificación final, además del comportamiento interno de la región y su comparación con el grupo de los 7 (G7). Los resultados muestran que no existe una variable que se correlacione con el índice de innovación y hay diferencias muy marcadas entre los dos grupos de estudio e incluso al interno de la región latinoamericana. Esto plantea retos muy importantes para mejorar este aspecto, pues también se demostró una correlación entre la innovación y la competitividad para los países industrializados que no se presenta para la región.
\end{abstract}

Palabras clave. Innovación, competitividad, Latinoamérica, industrializado, índice.

\begin{abstract}
It is common to associate innovation with the level of development and competitiveness of a country. The Global Innovation Index makes a classification in this area. For the Latin American countries, the production of patents was studied, the influence of the variables that the index considers in its evaluation and the final classification, as well as the internal behavior of the region and its comparison with the group of 7 (G7). The results show that there is no variable that correlates with the innovation index and that there are very marked differences between the two study groups and even within the Latin American region. This poses very important challenges to achieve an improvement in this aspect, since it also demonstrated a correlation between innovation and competitiveness for industrialized countries that does not present itself for the region.
\end{abstract}

Keywords. Innovation, competitiveness, Latin America, industrialized, index.

${ }^{1}$ Ingeniero Máster en Química Industrial y Administración de Empresas. Estudiante del doctorado en Ciencias Administrativas de la Universidad Estatal a Distancia, Costa Rica. Dirección electrónica: quesadaej@gmail.com 


\section{Quesada Espinoza}

\section{Introducción}

La relación entre la innovación, el crecimiento de la economía y el bienestar social fue propuesto por Adam Smith desde el siglo XVIII (Ketelhöhn y Ogliastri, 2013). En las últimas décadas, se ha brindado a este tema más importancia en los sectores académicos, empresariales y de gobierno. Esta relevancia se ha ligado a una correlación entre el nivel de innovación de los países y su competitividad, dentro del contexto de cambios estructurales como globalización y apertura de mercados (Moraleda, 2004).

Denton (1999) sostiene que la innovación ha sido siempre el centro de la competitividad. Los términos se escuchan muy frecuentemente cuando se analiza la situación de los países y su posibilidad de mejorar las condiciones de vida de sus habitantes, aunque no siempre se tiene claro a qué se refieren y cómo se miden. Por tal motivo, es preciso delimitar qué se entenderá por estos dos términos.

Existenmuchasdefinicionesdecompetitividad, por lo que es pertinente establecer una como referencia para el presente trabajo. Según el Foro Económico Mundial, se entiende por este concepto al "conjunto de instituciones, políticas y factores que determinan el nivel de productividad de un país (...) el nivel de productividad establece el nivel sostenible de prosperidad que puede ganar una economía" (Lombana y Rozas Gutiérrez, 2009, p. 10). Por su parte, la Comisión Europea sobre el tema la entiende como "altos y crecientes estándares de vida en una nación (o grupo de naciones) con el nivel más bajo posible de desempleo involuntario, sobre una base de sostenibilidad" (Aiginger, 2006, p. 64).

Otra de las aproximaciones al concepto, bastante reconocido por ser la base de un reporte global, es del Institute for Management and Development (IMD); esta se orienta no solo al beneficio de las personas que conforman una nación, sino que considera importante tener en cuenta a las empresas presentes(Lombana y Rozas Gutiérrez, 2009).

Desde la perspectiva de las empresas, la competitividad se interpreta como la capacidad de persuadir a clientes a escoger su oferta sobre las de las otras empresas o como la habilidad de mejorar continuamente el proceso (Feurer y Chaharbaghi, 1994). Desde las organizaciones se plantean opciones de gestión tecnológica (Ortega Gómez, 2000), las cuales pretenden enfrentar las altas tasas de obsolescencia en esta área. También, existen factores como los cambios de mercado, el corto ciclo de vida de los productos, rápidos cambios en las necesidades del mercado, entre muchos otros (Shepherd y Ahmed, 2000).

Rubio y Baz (2015) valoran el concepto de competitividad desde diferentes perspectivas. Desde el punto de vista empresarial, lo conciben como la capacidad de competir exitosamente en el mercado. En el caso de los políticos y analistas como un conjunto de indicadores; mientras que para los economistas y algunos empresarios, la competitividad se restringe al comparativo del valor de la mano de obra entre diferentes naciones. En el caso de Flores, Vega y Solís (2011, p. 745) entienden "por competitividad a la capacidad de una organización pública o privada, lucrativa o no, de mantener sistemáticamente ventajas que le permitan alcanzar, sostener y mejorar una determinada posición en el entorno socioeconómico".

Dada la amplia gama de aproximaciones al concepto, para efectos de este artículo, se considerará como definición de competitividad la que se refiere a la capacidad de las naciones para aumentar su productividad en procura de un beneficio de la sociedad, con la sostenibilidad ambiental como aspecto significativo. Esta definición incluye a las organizaciones y los individuos, los cuales se valoran como 
elementos que contribuyen a la consecución de un nivel competitivo determinado. Se entiende como una construcción en forma de pirámide, donde la base es constituida por los elementos mencionados, que aportan a la competitividad de una estructura mayor como lo es la nación.

Por otro lado, se encuentra la innovación. Para Martín, Ribeiro y Picazo (2012, p. 52) esta "no es un nuevo fenómeno que se está produciendo en las economías, sino que es un proceso inherente al desarrollo humano". Desde las sociedades primitivas, se han buscado los mecanismos para conseguir los medios de subsistencia. En la época más reciente, las empresas han aprovechado la innovación para elaborar productos más competitivos que permiten ampliar los mercados (Martín et al., 2012). La innovación entraña el propósito de mejorar la competitividad de las organizaciones incorporando tecnología y conocimientos nuevos (Albornoz, 2013).

Parece más complejo llegar a un consenso con respecto a la definición de este vocablo que para competitividad. Gee (1981) se refiere a la innovación como el proceso en el cual a partir de una idea, invención o reconocimiento de necesidad se desarrolla un producto, técnica o servicio útil y es aceptado comercialmente.

\section{Según Pavón y Goodman (citado por} Cilleruelo, 2007, p. 92), "es el conjunto de actividades inscritas en un determinado periodo de tiempo y lugar que conduce a la introducción con éxito en el mercado, por primera vez, de una idea en forma de nuevos o mejores productos, servicios o técnicas de gestión y organización". Esta definición considera que debe darse el cambio por primera vez, ello implícitamente manifiesta que sea nuevo.

Las dos definiciones previas enfocan el concepto a la incorporación al mercado. En el Manual de Oslo, se considera que "las innovaciones de producto implican cambios significativos de las características de los bienes o de los servicios. Incluyen ambos los bienes y los servicios enteramente nuevos y las mejoras significativas de los productos existentes. Las innovaciones de proceso son cambios significativos en los métodos de producción y de distribución" (OECD, 2007, p. 23). Esta última definición no restringe a la aceptación comercial de la innovación para considerarla como tal.

La innovación también se puede considerar "una serie de actividades científicas y tecnológicas, organizacionales, financieras y comerciales; acciones que, en potencia, transforman las fases productiva y comercial de las empresas" (Albornoz, 2013, p. 112). El mismo autor señala que es la base de la sociedad del conocimiento y también el motor de la globalización (Albornoz, 2013).

La innovación está más ligada al desarrollo de las organizaciones, en especial de las empresas. Es común encontrar formas de innovar en las compañías con el objetivo de mantener un nivel competitivo ante el mercado, por lo tanto, se encuentra abundante literatura en esta línea. Se podrían citar ejemplos variados de cómo han implementado innovaciones o de complicaciones que se tienen al llevarlas al mercado (Fernández González, 2013; Moraleda, 2004; Pomar Fernández, Rangel Magdaleno y Franco Zesati, 2014; Srivastava, Sultan y Chashti, 2017). Sin embargo, no debe dejarse de lado la innovación en el sector público, social y no gubernamental que no se documenta en el mismo grado que la anterior.

Existen diferentes formas de clasificar los tipos de innovación. Se pueden separar según el objeto de modificación o, como se detalla en la figura 1, donde la clasificación se efectúa a partir de la motivación (los dos tipos de la izquierda) y por su grado de impacto (los dos tipos de la derecha). 

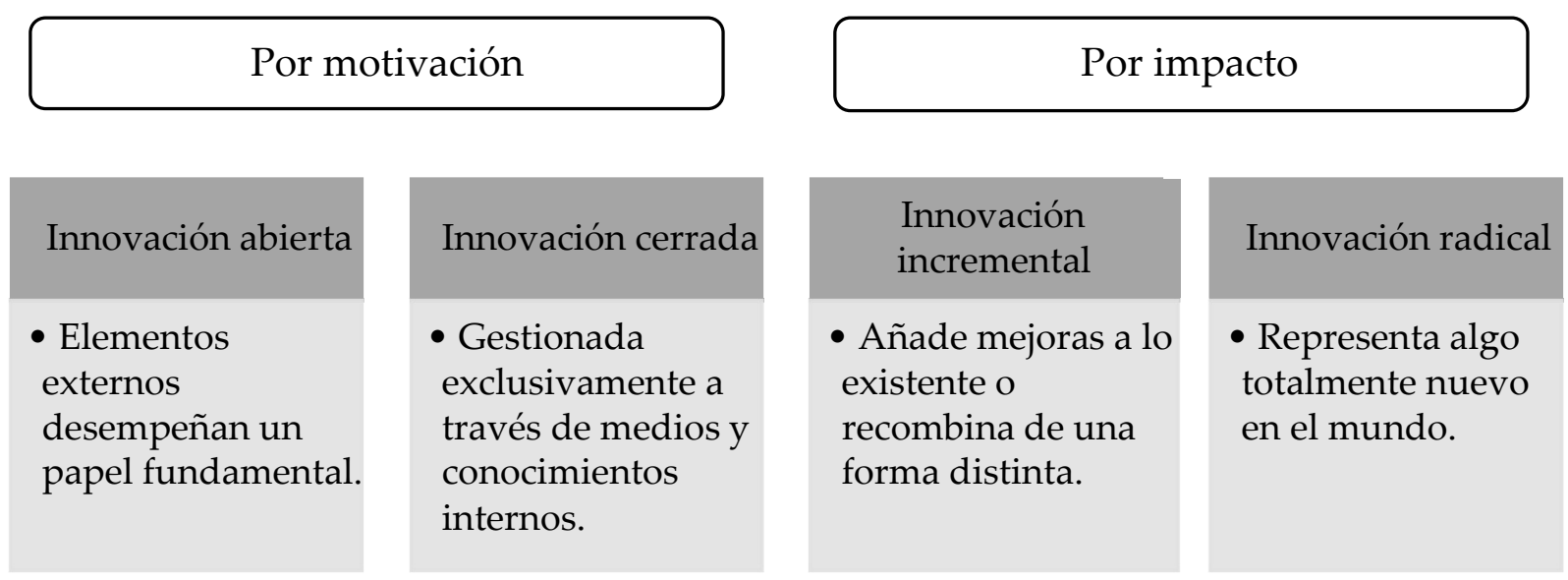

Figura 1. Descripción de cómo se clasifica la innovación según dos tipos de separación. Adaptado de “La gestión de la innovación y la competitividad desde la política pública en Costa Rica” por A. Sandoval Barraza, J. C. Bermúdez Mora, y J. Valverde Chaves, 2016, Revista 89.1, Enero-Junio, p. 128).

Resulta complicado establecer una definición consensuada para competitividad e innovación y es aún más difícil definir cómo medirlas. La Organización de Cooperación y Desarrollo Económico (OCDE) ha establecido una guía (Manual de Oslo) para lograr medir, de una forma medianamente homogénea, la innovación en todos sus países miembros, con criterios que sean representativos y ajustados a las características dispares de sus integrantes (OECD, 2007). El Manual de Oslo no se adapta de manera adecuada a las características de los países latinoamericanos, pero esto ya había sido contemplado en el área de la innovación tecnológica, promovido por varias instancias y que se denomina Manual de Bogotá (Jaramillo, Lugones y Salazar, 2001).

También existen dos metodologías que son las más aceptadas para efectuar las mediciones de innovación y competitividad a nivel de naciones. En primera instancia, se describirá el reporte anual elaborado por el Foro Económico Mundial (WEF, por sus siglas en inglés) que se fundamenta en una metodología de evaluación de lo que denominan pilares y se conoce como Reporte Global de Competitividad. Cada uno de los doce pilares (llamados variables en este trabajo) está conformado por una serie de puntos que se valoran; algunos de ellos son ponderados para evitar tendencias por efectos de tamaño de mercado, diversificación, población, entre otros (World Economic Forum, 2017).

Por otra parte, el World Intellectual Property Organization (WIPO) hace otra publicación anual en la que evalúa la innovación de las naciones por medio de una clasificación, el Índice Mundial de Innovación. Esta organización valora 7 puntos para obtener su índice. En la figura 2, se observan los pilares empleados en cada una de las mediciones. Si bien, en algunas, el nombre empleado coincide en ambas mediciones, los indicadores que se usan para evaluarlas difieren.

El Índice Mundial de Innovación se divide en dos subíndices: entrada y salida. En el primero, se incluyen los primeros cinco pilares mencionados en la figura 2, y en el segundo, los otros dos pilares. Los pilares de entrada capturan los elementos de 
la economía nacional disponibles para llevar a cabo las actividades innovadoras; los pilares de salida reflejan los resultados de las actividades innovadoras dentro de esa economía. En total, los pilares están conformados por 81 indicadores. El año específico para obtener los datos se enfocó en el sector alimentario, sin embargo, estos son funcionales para las comparaciones propuestas, pues se hacen de manera relativa y ponderada.

Otro elemento que puede usarse para valorar la innovación son las patentes registradas. La patente puede otorgarse a la invención o al descubrimiento de un proceso, máquina, fabricación o composición de materia nueva y útil, o a cualquier mejora nueva y útil de estos. La U. S. Patent and Trademark Office (USPTO) define los inventos "como conjuntos de capacidades tecnológicas, y la tecnología a su vez se define como la aplicación de la ciencia y la ingeniería al desarrollo de máquinas y procedimientos para mejorar las condiciones humanas, o al menos para mejorar su eficiencia" (Youn, Strumsky, Bettencourt, y Lobo, 2015, p. 2).

La generación de conocimiento patentable ha estado relacionada con la capacidad de las naciones de ser más innovadoras $\mathrm{y}$, por
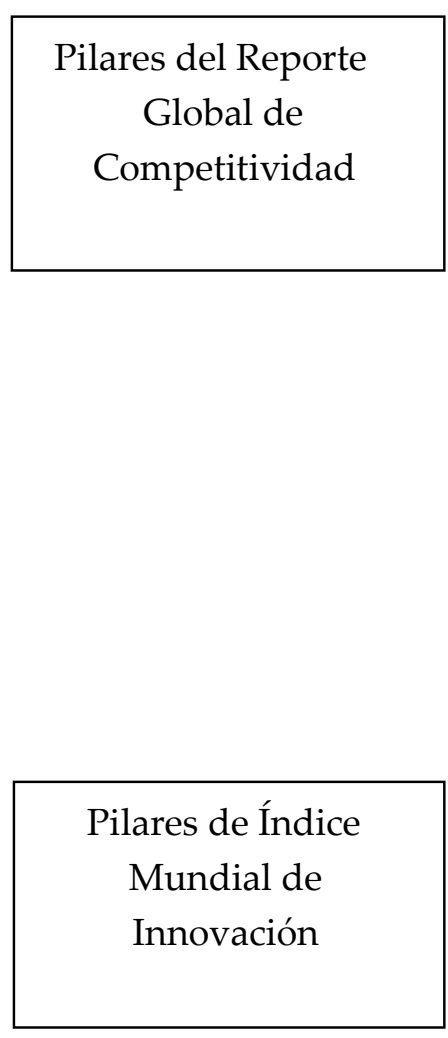
1. Instituciones
2. Infraestructura
3. Ambiente macroeconómico
4. Salud y educación primaria
5. Educación superior y técnica
6. Eficiencia del mercado de bienes
7. Eficiencia del mercado laboral
8. Desarrollo del mercado financiero
9. Preparación tecnológica
10.Tamaño de mercado
11. Sofisticación de los negocios
12. Innovación

1. Instituciones

2. Capital humano e investigación

3. Infraestructura

4. Sofisticación del mercado

5. Sofisticación de los negocios

6. Productos del conocimiento y la tecnología

7. Productos de creatividad

Figura 2. Pilares (variables) considerados por el Reporte Global de Competitividad y el Índice Mundial de Innovación. 


\section{Quesada Espinoza}

medio de esto, lograr mayor competitividad. Sin embargo, los datos de patentes pueden no capturar la innovación que ocurre, por ejemplo, en países sin leyes de patentes, las personas inventoras confían en mecanismos alternativos para proteger su propiedad intelectual (Moser, 2013).

La mayoría de estos mecanismos alternos para proteger las invenciones no cuentan con registros como el del caso de las patentes, por esta razón se hace una relación con el número de patentes registradas y no con otros mecanismos de protección. En virtud de que la USPTO es la oficina con mayor registro de patentes globalmente, es usual emplearla como fuente de datos, con la conciencia del sesgo que genera en el caso de la información de los Estados Unidos.

Teniendo como premisa que la competitividad está relacionada con la innovación, en las próximas páginas se compararán los datos para demostrar o refutar la afirmación anterior. Para ello, se analizará el comportamiento de las patentes, se buscará establecer cómo impactan las variables medidas en la innovación, y la relación entre innovación y competitividad en los países latinoamericanos y su comparación con los países miembros del denominado grupo de los 7 (G7).

\section{Metodología}

Los datos fueron tomados de tres fuentes diferentes. Los referentes a patentes registradas en los Estados Unidos se obtuvieron de la oficina de patentes de ese país (U. S. Patent and Trademark Office, 2017); a su vez, fueron procesados para obtener indicadores que muestren las diferencias o similitudes de los países estudiados.

La segunda fuente de datos son los índices más importantes en el tema de innovación y competitividad: el Índice Mundial de Innovación
2017 y el Reporte Global de Competitividad 2017-2018 (Cornell University, INSEAD y WIPO, 2017; World Economic Forum, 2017). Ambos documentos son de acceso libre y los datos sin procesar están disponibles en ambas fuentes. Para el año 2017, los datos del Índice Mundial de Innovación se enfocaron en el sector alimentario; sin embargo, son funcionales para las comparaciones propuestas, pues se hacen de manera relativa y ponderada.

A partir de la información, se eligieron los países latinoamericanos, a saber: Jamaica, Trinidad y Tobago, Chile, Uruguay, Costa Rica, Panamá, Perú, El Salvador, República Dominicana, Guatemala, Honduras, Paraguay, Ecuador, México, Colombia, Argentina y Brasil, que se reflejaban en los dos reportes, y los países del G7: Canadá, Francia, Japón, Italia, Alemania, Reino Unido y Estados Unidos.

Los datos se trabajaron estadísticamente procurando relaciones entre la innovación y competitividad. Se efectuaron regresiones lineales para establecer la relación entre las variables medidas en el Reporte de Competitividad Global y el índice de innovación reportado, empleando para decidir el R2 ( $\mathrm{r}$ cuadrado). El nivel de confianza con el que se trabajó fue del $95 \%$.

También se procuró establecer cómo las variables medidas en el Índice Mundial de Innovación se relacionaban o no en los grupos de países señalados anteriormente y entre estos. Para este objetivo, se usó el análisis de componentes principales (PCA, por sus siglas en inglés). Los valores de las siete variables consideradas en el Índice Mundial de Innovación fueron introducidos en el paquete estadístico empleado, entendiendo que son independientes entre sí.

El paquete estadístico utilizado fue SPSS en su versión 23.0.0.0 para Windows en 64 bit. Los 
datos originales fueron trabajados para ajustarlos a los requisitos de ingreso al software señalado.

\section{Resultados y discusión}

Con el fin de analizar las características de los grupos de estudio definidos (los países latinoamericanos y el grupo de los siete), para establecer similitudes o diferencias entre ellos y a lo interno de cada uno, se trabajó comparando las patentes aprobadas por la oficina respectiva de los Estados Unidos. Otra herramienta fue la regresión lineal con el objetivo de identificar cuáles de las variables medidas se correlacionan fuertemente con el índice de innovación $\mathrm{o}$, al contrario, no presentan correlación. Además, se estudió cómo se agrupan la innovación y la competitividad en un gráfico de dispersión para identificar patrones. Finalmente, se analizaron los componentes principales para cada uno de los grupos con las siete variables dispuestas por el Índice Mundial de Innovación, mencionadas en la figura 2, y se compararon los dos grupos.

\section{Producción de patentes}

Partiendo de la hipótesis que la innovación está asociada de manera estadísticamente significativa con la competitividad de un país, es habitual relacionar el número de patentes con la capacidad de un país para desarrollarse en un ambiente global con alta competitividad. El mercado más atractivo que insta a solicitar una patente es el estadounidense. La oficina de patentes de los Estados Unidos maneja la información al respecto, desde el año 1977 al 2015, en línea. Los registros aprobados no solo se generan dentro del territorio estadounidense, sino que cualquier persona física o jurídica del mundo puede realizar el procedimiento respectivo de solicitud. En la tabla 1, se detallan las patentes otorgadas por la Oficina de Patentes de los Estados Unidos desde 1977.
Es de esperar que los datos, en términos absolutos, sean dominados por el país al cual pertenece la oficina. Sin embargo, se nota una gran diferencia entre los latinoamericanos y los del G7. Una forma de medirlo de manera relativa es el número de patentes por cada 100000 habitantes, también denominado coeficiente de invención (Fernández, 2017). En este indicador los valores del G7 se encuentran en el rango de las centenas y los de América Latina no alcanzan el rango de las decenas. No obstante, se destacan dos países con una cantidad de patentes que supera sustancialmente al promedio: Costa Rica $(7,0)$ y Uruguay $(4,7)$.

El dato alentador es que el crecimiento en los periodos señalados mantiene una razón muy similar, destacan Brasil y Chile que superan el valor de 3. Claro está que es imposible recuperar el tiempo perdido y que la tasa de crecimiento debería ser exponencial para lograr el número de patentes de los países del G7.

Aunque los datos relativos de crecimiento no son negativos y muestran que Latinoamérica se encuentra en un nivel similar al de los G7, es preciso aumentar la producción de patentes que tengan impacto global. Es probable que muchos de los países cuenten con mayor cantidad de patentes otorgadas a lo interno de su territorio, las cuales no se contabilizan, pero que, en su mayoría, es poco probable que tengan una relevancia sustancial. Es preocupante esta baja cantidad de patentes, pues, para el 2011, el producto interno bruto de Latinoamérica representó el 8,7 \% global, pero solo produjo el 0,17 \% de las patentes desde 1977 (Ketelhöhn y Ogliastri, 2013).

Correlación entre variables, innovación $y$ competitividad

El número de patentes no es el único factor importante dentro de las mediciones de innovación de un país. Esto lo conocen los 


\section{Quesada Espinoza}

Tabla 1.

Producción de patentes registradas en los Estados Unidos en Latinoamérica y el grupo de los 7

\begin{tabular}{|c|c|c|c|}
\hline País & $\begin{array}{c}\text { Patentes } \\
\text { desde } 1977\end{array}$ & $\begin{array}{c}\text { Patentes } / 100000 \\
\text { habitantes }^{\mathrm{a}}\end{array}$ & $\begin{array}{l}\text { Crecimiento } \\
(77-02)-(03-15)^{\mathrm{b}}\end{array}$ \\
\hline \multicolumn{4}{|c|}{ Latinoamérica } \\
\hline Argentina & 1512 & 3,5 & 2,2 \\
\hline Brasil & 4116 & 2,0 & 3,3 \\
\hline Chile & 598 & 3,4 & 3,9 \\
\hline Colombia & 348 & 0,7 & 2,4 \\
\hline Costa Rica & 338 & 7,0 & 2,6 \\
\hline República Dominicana & 55 & 0,5 & 2,6 \\
\hline Ecuador & 81 & 0,5 & 2,4 \\
\hline El Salvador & 26 & 0,4 & 1,7 \\
\hline Guatemala & 55 & 0,3 & 1,8 \\
\hline Honduras & 24 & 0,3 & 1,5 \\
\hline Jamaica & 62 & 2,2 & 2,7 \\
\hline México & 3006 & 2,4 & 2,4 \\
\hline Panamá & 50 & 1,3 & 2,1 \\
\hline Paraguay & 12 & 0,2 & 2,0 \\
\hline Perú & 112 & 0,4 & 1,8 \\
\hline Trinidad y Tobago & 81 & 6,0 & 2,1 \\
\hline Uruguay & 161 & 4,7 & 1,6 \\
\hline \multicolumn{4}{|c|}{ Grupo de los 7} \\
\hline Alemania & 365627 & 447,6 & 2,0 \\
\hline Canadá & 123904 & 345,6 & 2,4 \\
\hline Estados Unidos & 3030080 & 944,3 & 2,1 \\
\hline Francia & 139866 & 209,9 & 1,9 \\
\hline Italia & 62148 & 102,3 & 2,0 \\
\hline Japón & 1069394 & 841,1 & 2,3 \\
\hline Reino Unido & 140227 & 215,3 & 2,0 \\
\hline \multicolumn{4}{|c|}{$\begin{array}{l}\text { Notas: }{ }^{\mathrm{a}} \text { Datos de población tomados del Banco Mundial para el año } 2015 \\
{ }^{\mathrm{b}} \text { Relación estimada como la división de las patentes otorgadas de } 1977 \\
\text { a } 2002 \text { entre las de } 2003 \text { a } 2015 .\end{array}$} \\
\hline
\end{tabular}

Fuente:Adaptado de "U.S. Patent Counts By Country / State and Year All Patents, All Types Patent Counts By Country / State and Year All Patents, All Types", por Patent and Trademark Office, 2017, recuperado noviembre 11, 2017, de https://www.uspto.gov/web/offices/ac/ido/oeip/taf/cst_all.htm y “Total Population”, World Bank Group, 2017, recuperado noviembre 10, 2017, de https://data.worldbank.org/indicator/SP.POP.TOTL 
países y las organizaciones siguen el tema. El Indice Mundial de Innovación ha considerado siete variables para tratar de evaluar de manera más objetiva (ver figura 2). Después de realizar una regresión, empleando como variable independiente cada una de las siete variables y como dependiente el Indice Mundial de Innovación, se comprobó que no existe una correlación significativa entre ninguna de las variables y la innovación (datos estadísticos no presentados). Por el contrario, se identificó que la variable "Sofisticación de mercado" no se correlaciona con el índice de innovación, con un R2 de 0,020 en el caso de los países latinoamericanos. El grupo G7 también presentó una variable que no se correlaciona, en este caso "Infraestructura", con un valor de R2 de 0,065. Claramente, no existe relación entre estas variables y el valor del índice.

$\mathrm{Si}$ se considera que, en Latinoamérica, los mercados no presentan un nivel de sofisticación importante, es de esperar que no exista correlación. Mucha de la innovación se produce en sectores que son relevantes para la región, pero no necesariamente para los países industrializados. La no correlación de la infraestructura e innovación en el grupo G7 puede darse por el alto nivel de manejo de información y tecnologías que evalúa esta variable y algunas posiciones en estos apartados en el índice no son las más altas.

Con el propósito de comprobar la hipótesis de que la innovación está relacionada directa y positivamentecon la competitividad delos países, se efectuó una regresión lineal considerando como variable independiente el Índice Mundial de Innovación y como variable dependiente la competitividad para el G7 y Latinoamérica. Los resultados muestran una mejor correlación en el primer grupo con un valor de R2 de 0,85 en comparación con un valor de 0,56 para el segundo. En los países industrializados, la innovación, al menos en el campo alimentario, es un buen predictor de la competitividad del país, mientras que para los países de América Latina resulta no serlo. La línea que debe seguir nuestra región es hacia el apoyo a la innovación, ello implica una inversión dirigida hacia la investigación y el desarrollo económico.

Por otro lado, estudiar los datos de las diferentes variables para establecer patrones que permitan identificar similitudes entre los países resulta un trabajo complejo. Al graficar los resultados de competitividad contra el índice de innovación para los países latinoamericanos bajo estudio, se observa que se divide en dos grupos (ver figura 3). Esta metodología fue empleada para analizar la relación existente en la Unión Europea, obteniendo separaciones en grupos bien definidos también (Petrakis, Kostis y Valsamis, 2015).

Uno de los grupos (ubicado a la derecha de la figura 3) incluye los países que desde hace varios años han venido mostrando buenos registros en términos de competitividad. Jamaica es el único que parece anómalo en este agrupamiento. En el otro grupo, es interesante encontrar a Argentina junto con países que parecen tener menos capacidad innovadora y de competencia. Esta figura muestra cómo, incluso en países que presentan características, hasta cierto punto similares, se encuentran agrupaciones que son imposibles de ver con los datos sin procesar.

\section{Análisis de componentes principales}

Las agrupaciones no solo se encuentran en la comparación de diferentes índices; por eso, con el propósito de observar cómo se agrupan los diferentes países, se procedió a analizar por componentes principales. En el caso de Latinoamérica, se observar en la figura 4 cómo los dos primeros componentes muestran una agrupación. Cada uno de los conglomerados se identifica mediante diferentes colores. El grupo 


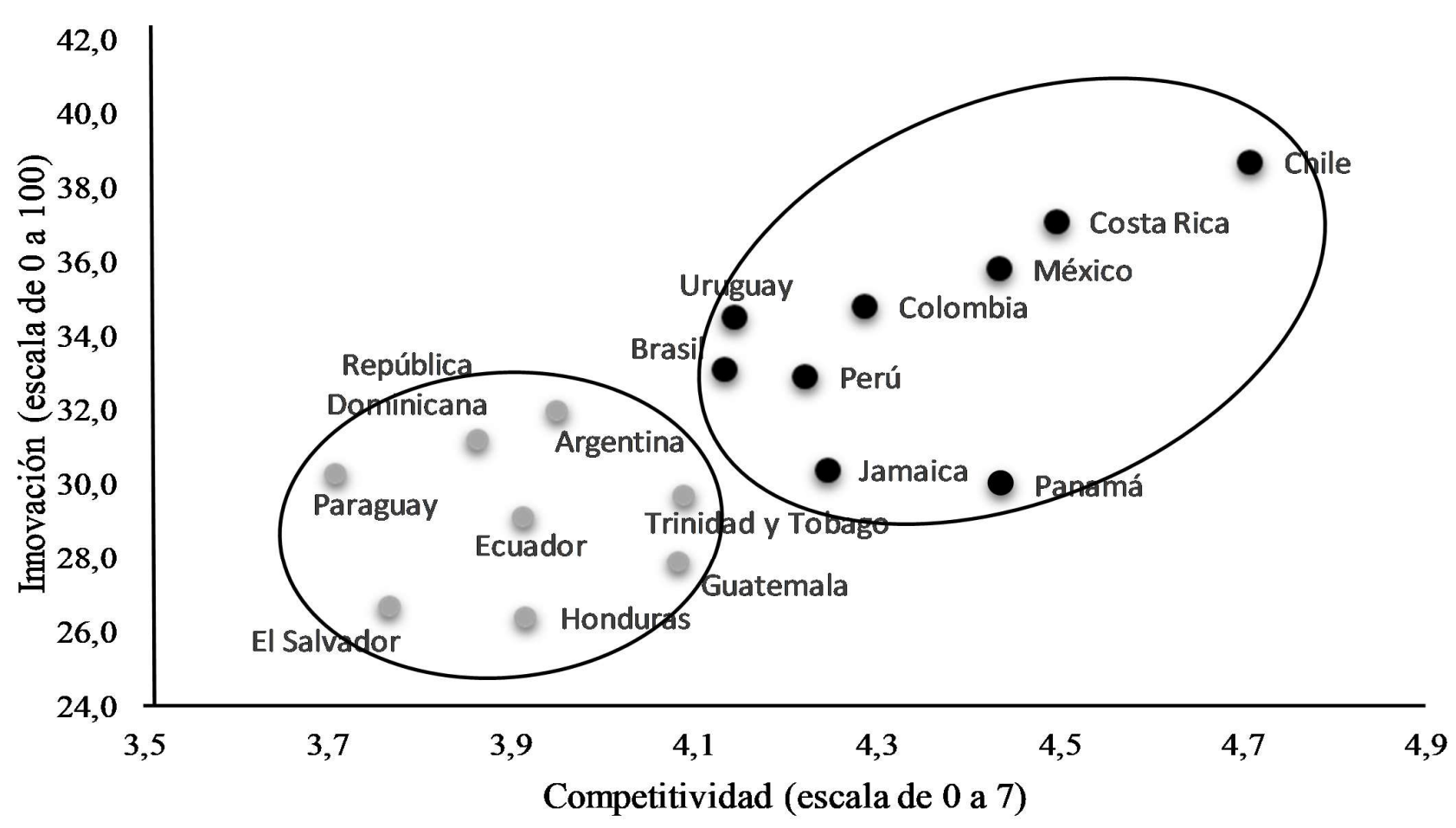

Figura 3. Representación de cómo se agrupan los países latinoamericanos, según su puesto en el Índice Mundial de Innovación y de Competitividad Global.

superior izquierdo se caracteriza por tratarse de dos países del Caribe, por eso es de esperar que tengan condiciones similares. Sin embargo, República Dominicana no está cerca de ellos y se desplaza al grupo inferior izquierdo, este país tiene acuerdos comerciales con los países centroamericanos ubicados en este mismo conglomerado y se ratifican sus similitudes. Paraguay y Ecuador tienen características de innovación parecidas a las de los países mencionados.

Los grupos del lado derecho tienen más aspectos semejantes. El conglomerado inferior está constituido por países con economías más grandes, con los mercados más importantes de la región; aunque se percibe cierta dispersión, principalmente por la posición de Argentina.
Esto es consistente con lo observado en la figura 3 , donde se ubica en el grupo inferior, a diferencia de Brasil, Colombia y México. El último grupo es el superior derecho, donde se encuentra Costa Rica; está constituido por países caracterizados por estabilidad relativa en sus sistemas democráticos y que buscan apertura al comercio internacional. Ambos pueden ser factores importantes para promover la innovación hacia el futuro. Además, según el Índice de Prosperidad, estos mismos países se ubican como los mejores de la región (Chinchetru, 2017).

Cuando se aplica la misma metodología para el G7, no se observa agrupación alguna (gráfico no presentado). Cada uno de los países se ubica separadamente indicando que las siete variables empleadas por el Índice Mundial de 


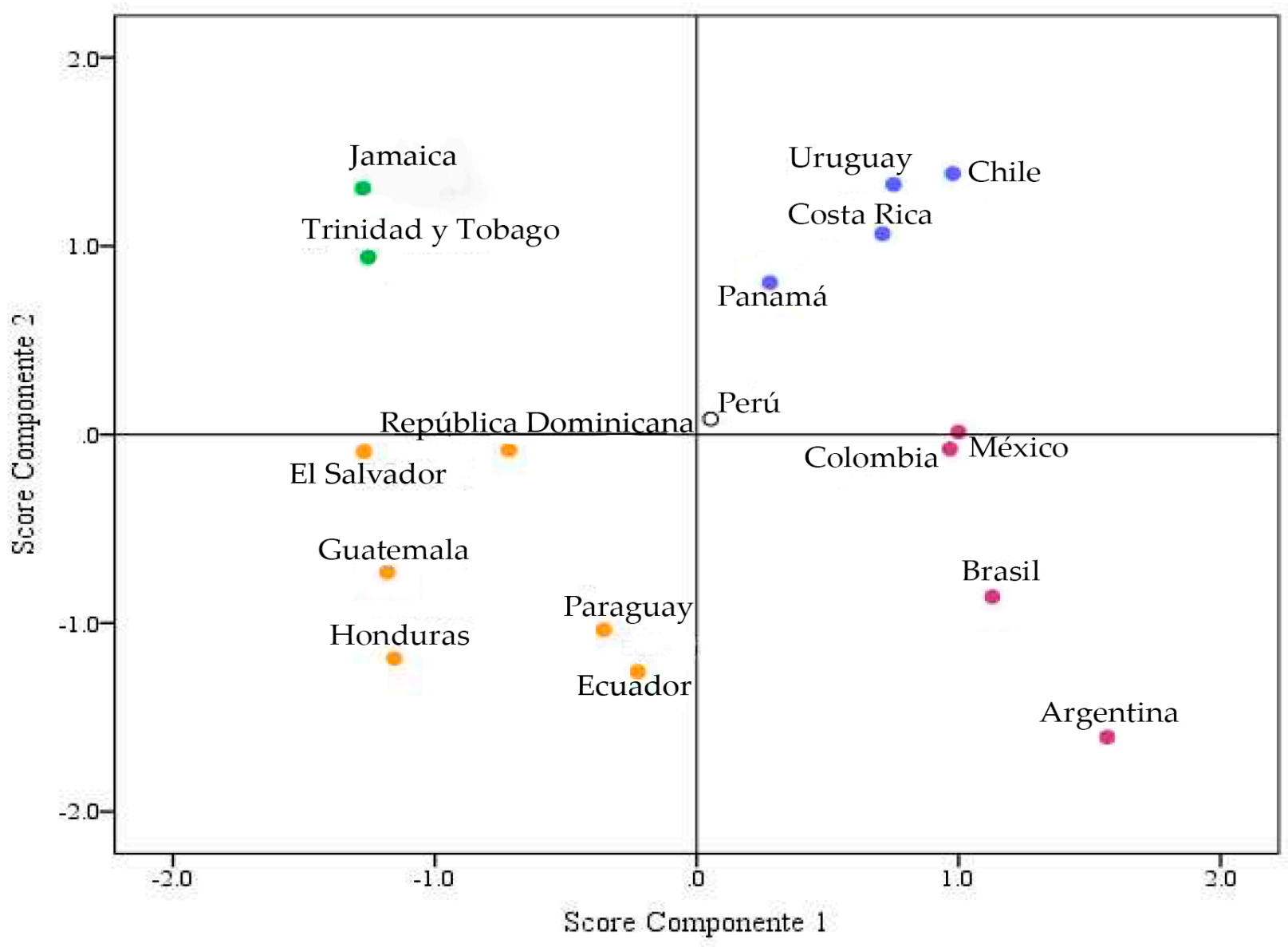

Figura 4. Representación de los scores del análisis de componentes principales para los países latinoamericanos estudiados.

Innovación no se comportan de manera similar en el bloque. A pesar de ser los denominados países industrializados un grupo que comparte características, los modelos de desarrollo no son iguales. Esto se reafirma en la tabla 1, donde se expone una disparidad en las patentes registradas en los Estados Unidos.

Las comparaciones entre los países pertenecientes a los dos grupos definidos como objeto de estudio muestran comportamientos muy distintos al evaluar las variables que mide el Índice Mundial de Innovación. Con el fin de definir cuán similares o diferentes son los grupos entre sí, se procedió a analizar los componentes principales a ambos grupos en conjunto. Los resultados (figura 5) evidencian una separación clara entre los países latinoamericanos y el G7. Esto no parece especialmente llamativo, excepto por la posición de Italia en un espacio intermedio entre los dos conglomerados; lo cual significa que se parece menos al G7 y más al otro grupo de países. Esto es consistente con lo detallado en la tabla 1, donde Italia es el menor productor de patentes por cada 100000 habitantes, el más bajo de los países industrializados. No es el único factor, pero justifica parcialmente el comportamiento mencionado. 


\section{Quesada Espinoza}

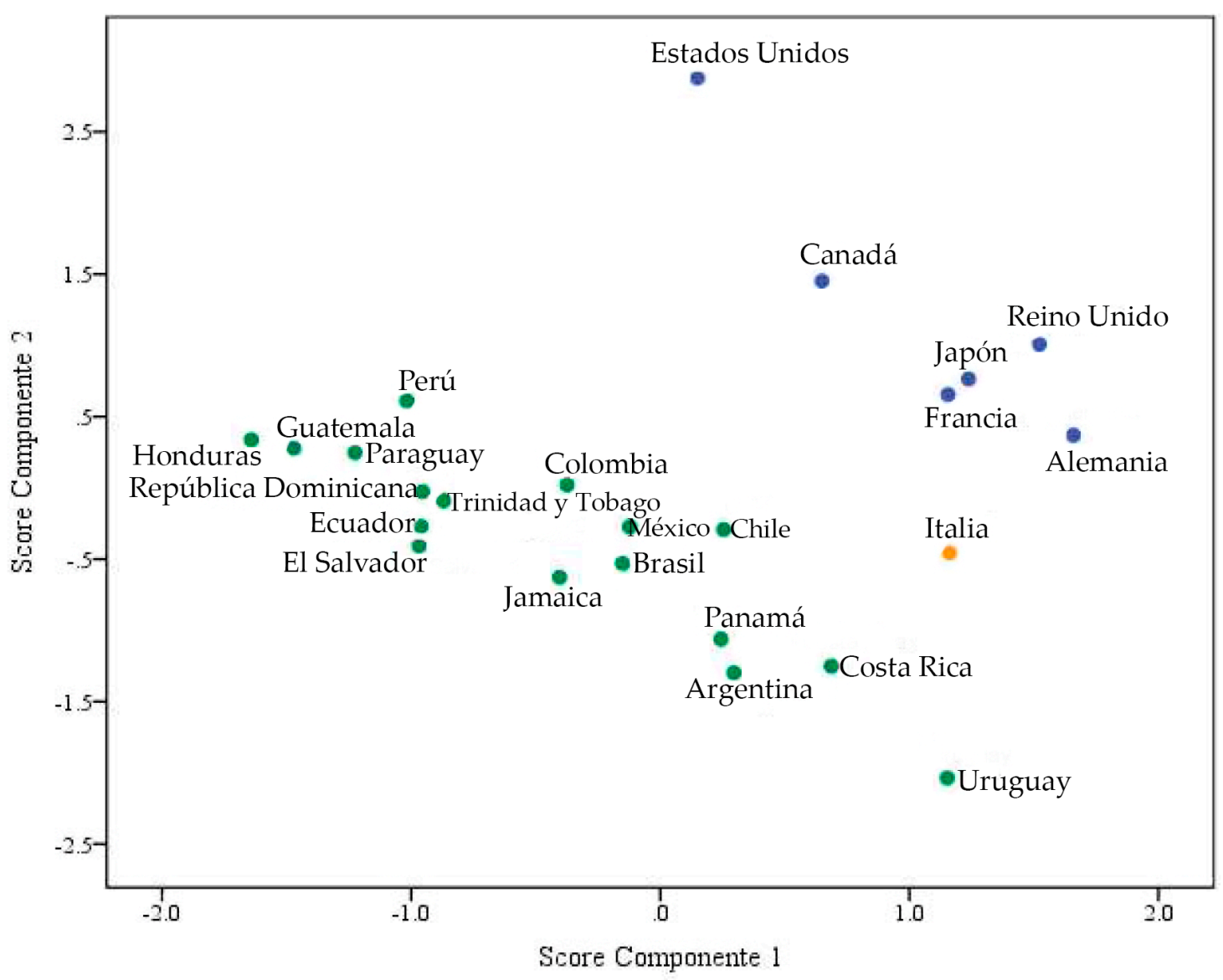

Figura 5. Representación de los scores del análisis de componentes principales para los países latinoamericanos estudiados y grupo de los 7.

\section{El reto de la innovación en Latinoamérica}

La innovación en la región de Latinoamérica se relaciona con la competitividad, pero no es promovida significativamente por ninguna de las variables que forman parte del Índice Mundial de Innovación, resultado que podía ser previsible por el enfoque en el aspecto alimentario de este índice en el 2017. Se observan diferencias significativas entre la región y los países industrializados, que son representados por el G7. Existen pocas similitudes en aspectos como la inversión en investigación y desarrollo, pues Latinoamérica invierte menos del $1 \%$ del producto interno bruto, mientras que Asia y Europa rondan entre el 2 \% y 2,5\% (Olavarrieta y Villena, 2014). Con esta brecha es difícil que los países logren aumentar su competitividad y se acerquen a los países en el percentil superior.

Asimismo, se considera que, en algunos países (especialmente pequeños), no existe un aporte importante de una multinacional que invierta en innovación, Chile y Costa Rica son ejemplos donde se manifiesta esto (Crespi y Zuñiga, 2012). Aunado a ello, existen escasas políticas públicas que promuevan la inversión. Son pocos los casos donde se han tomado decisiones por parte de los gobiernos que impacten directamente la innovación. También, se plantea que políticas innovadoras pueden mejorar la competitividad, por ejemplo, la apertura del mercado de energía renovables en Brasil (Bradshaw, 2016). 
Según Llisterri y Pietrobelli (2011), Latinoamérica se ha olvidado del sistema regional de innovación (SRI). El concepto se basa en la premisa de que el proceso de innovación es esencialmente social, dado que conlleva el involucramiento de las empresas, las agencias promotoras de la innovación, las instituciones académicas y los centros de investigación. El proceso no se realiza solo al interior de las organizaciones y como el conocimiento está asociado a las personas hace que el componente territorial, sus redes y sus capacidades influyan en su desempeño innovador.

Lo anterior implica que cada país de la región debe encontrar la forma adecuada de amalgamar a todos los participantes para obtener el desarrollo de la innovación, el cual se refleje en las otras variables que conforman la medición de competitividad. No se pueden ver solo números fríos que miden volumen y no necesariamente calidad. Sin embargo, en el caso de América Latina, no se muestran buenos indicadores en las mediciones numéricas ni en los resultados. Este es el verdadero reto de la región: asociar la innovación con un desarrollo que le brinde a sus pobladores condiciones buenas de vida.

\section{Conclusiones}

La región latinoamericana no muestra coeficientes de invención altos, esto significa que no puede sustentar su competitividad en ese aspecto, lo que habitualmente se relaciona con niveles elevados de innovación. Se nota un crecimiento sostenido que está al ritmo de los países industrializados, pero, en valores absolutos, tiene grandes diferencias.

El Índice Mundial de Innovación no presenta correlación significativa con ninguna de las variables que se emplean para el cálculo de este índice. Por tanto, tener un desempeño alto en cualquiera de estas no ayuda a mejorar de manera sustancial la ubicación en el índice.
Por el contrario, se identificaron variables que prácticamente no tienen relación, tanto para los países industrializados como para los de América Latina. Por tanto, la desmejora en estas variables no se vería reflejada en la ubicación en el índice. Sin embargo, si se considera una correlación entre la posición en innovación y la competitividad del país para los industrializados, se concluye que la innovación es relevante para lograr mejores puestos en este aspecto y, por ende, las posiciones de los países latinoamericanos.

No solo se identifican diferencias entre los dos grupos estudiados, también dentro de América Latina se observan subgrupos que denotan las disparidades entre los países a pesar de la cercanía geográfica. Esto refleja resultados diferentes según los modelos de desarrollo que se han adoptado y su impacto en la innovación. Aún existe mucha distancia entre los países y será difícil acortar sustancialmente esa brecha a corto o mediano plazo.

Lainnovación, vista comparativamente entrela misma región, muestra diferencias importantes, lo que evidencia hasta cuatro grupos separados en su nivel en este aspecto. Si se compara con los países industrializados, se observa una marcada diferencia, esto indica que existe una brecha entre los dos grupos. No se aprecia que ningún país latinoamericano pueda acercarse a los industrializados y, por el contrario, se concluye que Italia está más próxima a nuestra región.

La región debe enfocarse en un desarrollo que promueva innovar, donde todos los actores que se involucren estén presentes. Empresas, instituciones académicas, centro de investigación y gobierno tienen que trabajar en conjunto para hacer que la investigación se torne en innovaciones que produzcan resultados, los cuales se reflejen en el nivel de vida de las personas. 


\section{Quesada Espinoza}

\section{Referencias}

Aiginger, K. (2006). Revisiting an evasive concept: Introduction to the special issue on competitiveness. Journal of Industry, Competition and Trade, 6(2), 63-66. https://doi. org/10.1007/s10842-006-9471-x

Albornoz, M. (2013). Innovación, equidad y desarrollo latinoamericano Innovation, equity and Latin American development. ISEGORÍA, 48(Enero-junio), 111-126. https:// doi.org/10.3989/isegoria.2013.048.06

Bradshaw, A. (2016). Regulatory change and innovation in Latin America: The case of renewable energy in Brazil. Utilities Policy, 49, 156-164. https://doi.org/10.1016/j. jup.2017.01.006

Chinchetru, A. (2017, abril 19). Chile, Costa Rica, Uruguay y Panamá son los países más libres y prósperos de América Latina. Recuperado noviembre 23, 2017, de https://alnavio.com/ chile-costa-rica-uruguay-y-panama-son-lospaises-mas-libres-y-prosperos-de-americalatina

Cilleruelo, E. (2007). Compendio de definiciones del concepto «innovación» realizadas por autores relevantes: diseño híbrido actualizado del concepto. Dirección y Organización, 34(36), 91-98. Recuperado de http://revistadyo.es/ index.php/dyo/article/viewArticle/20

Cornell University, INSEAD, y WIPO. (2017). The Global Innovation Index 2017. (S. Dutta, B. Lanvin, y S. Wunsch-Vincent, Eds.). Genova: World Intellectual Property Organization.

Crespi, G., \& Zúñiga, P. (2012). Innovation and Productivity: Evidence from Six Latin American Countries. World Development, 40(2), 273-290. https://doi.org/10.1016/j. worlddev.2011.07.010
Denton, K. (1999). Gaining competitiveness through innovation. European Journal of Innovation Management, 2(2), 82-85.

Fernández González, J. P. (2013). Alpina: un caso de innovación para la competitividad. Revista de Ingeniería, 38(Enero-junio), 78-85. Recuperado de http://search.ebscohost.com/ login.aspx?direct=true \&db=a9h\&AN=9153127 5\&lang=es\&site=ehost-live

Fernández, V. (2017). The finance of innovation in Latin America. International Review of Financial Analysis, 53, 37-47. https://doi.org/10.1016/j. irfa.2017.08.008

Feurer, R., \& Chaharbaghi, K. (1994). Defining Competitiveness. Management Decision, 32(2), 4958. https://doi.org/10.1108/00251749410054819

Flores, M. V., Vega, A., \& Solís, M. M. (2011). Factores de contingencia que inciden en la profesionalización y competitividad de las empresas familiares del sector industrial de Tijuana, B. C., México. In Global Conference on Business and Finance Proceedings (pp. 743-753).

Gee, S. (1981). Technology transfer, Innovation $\mathcal{E}$ International Competitiveness. New York: Wiley and Sons.

Jaramillo, H., Lugones, G., \& Salazar, M. (2001). Manual de Bogotá. RICYT. Bogotá, Colombia.

Ketelhöhn, N., \&Ogliastri, E. (2013). Introduction: innovation in Latin America. Academia Revista Latinoamericana de Administración, 26(1), 12-32. https://doi.org/10.1108/ARLA-05-2013-0037

Llisterri, J. J., \& Pietrobelli, C. (2011). Introducción. En J. J. Llisterri y C. Pietrobelli (Eds.), Los sistemas regionales de innovación en América Latina (p. 124). Washington: Banco Interamericano de Desarrollo.

Lombana, J., \& Rozas Gutiérrez, S. (2009). Marco analítico de la competitividad: Fundamentos para el estudio de la competitividad regional. Pensamiento y Gestión, 26, 38. Recuperado de 
http://rcientificas.uninorte.edu.co/index.php/ pensamiento/article/viewFile/854/499

Martín, M. Á. G., Ribeiro, D., \& Picazo, M. T. M. (2012). Innovación y crecimiento económico: Factores que estimulan la innovación. Cuadernos de Gestión, 12 (SUPPL. PECIALISSU), 51-58. https://doi.org/10.5295/cdg.110309mg

Moraleda, A. (2004). La innovación, clave para la competitividad empresarial. Universia Business Review, Primer Tri, 128-136. https:// doi.org/10.1016/j.cede.2011.07.004

Moser, P. (2013). Patents and innovation: Evidence from economic history. Journal of Economic Perspectives, 27(1), 23-44. https://doi. org/10.1257/jep.27.1.23

Organización para la Cooperación y el Desarrollo Económicos (OECD). (2007). Manual de Oslo. Madrid: Grupo Tragsa. https:// doi.org/10.1787/9789264065659-es

Olavarrieta, S., y Villena, M. G. (2014). Innovation and business research in Latin America: An overview. Journal of Business Research, 67(4), 489-497. https://doi.org/10.1016/j. jbusres.2013.11.005

Ortega Gómez, J. A. (2000). Gestión de la tecnología, innovación y competitividad empresarial. Ingeniería y Competitividad, 2, 7-11. Recuperado de http://search.ebscohost. $\mathrm{com} / \operatorname{login}$.aspx?direct $=$ true $\& \mathrm{db}=\mathrm{a} 9 \mathrm{~h} \& \mathrm{AN}=20$ 432909\&lang $=$ es\&site $=$ ehost-live

Petrakis, P. E., Kostis, P. C., y Valsamis, D. G. (2015). Innovation and competitiveness: Culture as a long-term strategic instrument during the European Great Recession. Journal of Business Research, 68(7), 1436-1438. https:// doi.org/10.1016/j.jbusres.2015.01.029

Pomar Fernández, S., Rangel Magdaleno, J. A., y Franco Zesati, R. E. (2014). La influencia de las barreras a la innovación que limitan la competitividad y el crecimiento de las pymes manufactureras. Administración y Organizaciones, 17(33), 33-57.

Rubio, L. \& Baz, V. (2015). El poder de la competitividad. México: Fondo de Cultura Económica. Recuperado de http://www. cidac.org/esp/uploads/1/Competitividad_ FINAL-Agosto_2004.pdf

Shepherd, C., \& Ahmed, P. P. K. (2000). From product innovation to solutions innovation: a new paradigm for competitive advantage. European Journal of Innovation Management, 3(2), 100-106. Recuperado de http://www.emeraldinsight.com/journals. htm?articleid $=872485 \&$ show $=$ abstract $\% 5 C$

Srivastava, S., Sultan, A., y Chashti, N. (2017). Influence of innovation competence on firm level competitiveness: an exploratory study. Asia Pacific Journal of Innovation and Entrepreneurship, 11(1), 63-75. https://doi. org/10.1108/APJIE-04-2017-021

U. S. Patent and Trademark Office (2017). Patent Counts By Country / State and Year All Patents, All Types Patent Counts By Country I State and Year All Patents, All Types. Recuperado noviembre 11, 2017, de https:// www.uspto.gov/web/offices/ac/ido/oeip/ taf/cst_all.htm

World Economic Forum (2017). The Global Competitiveness Report 2017-2018. (K. Schwab, Ed.) (Vol. 5). Genova: World Economic Forum. https://doi.org/92-9504435-5

Youn, H., Strumsky, D., Bettencourt, L. M. A., y Lobo, J. (2015). Invention as a combinatorial process: evidence from US patents. Journal of The Royal Society Interface, 12, 1-8. http:// dx.doi.org/10.1098/rsif.2015.0272

Recibido: 22 de mayo de 2018 Aceptado: 2 de noviembre de 2019 